\title{
Viruses are models for embracing diversity
}

\author{
The first demonstration that RNA virus populations are groups of closely related sequences, proposed to behave as \\ a quasispecies, was published 40 years ago this month, marking the beginning of a fundamental change in how we \\ understand RNA virus dynamics, pathogenesis and response to therapy.
}

T his month marks 40 years since the publication of a study in Cell reporting that populations of the bacteriophage $\mathrm{Q} \beta$ are not genetically homogeneous, but rather a collection of distinct but closely related RNA sequences that arise as a consequence of the high error rate during replication ${ }^{1}$. The study, led by Esteban Domingo while in the laboratory of Charles Weissmann, proposed that such a population structure was an example of quasispecies, a concept that had been recently developed by biophysical chemists Manfred Eigen and Peter Schuster to explain the error-prone self-organization and evolution of macromolecules present at the origin of life ${ }^{2}$. To mark the anniversary of this seminal study in viral evolution, this issue features an interview with Esteban Domingo, in which he discusses how the group came to these results, how they were received, their implications and his vision for the future of the quasispecies field.

In the intervening 40 years, and although exactly how the quasispecies theory can be applied to RNA viruses has been the subject of some debate ${ }^{3,4}$, the concept has provided an important framework to understand the population structure and dynamics of numerous RNA viruses, including HIV, hepatitis $C$ virus, influenza virus and flaviviruses ${ }^{5}$. Viral pathogenesis was previously thought to be unrelated to virus variability and evolution — indeed, RNA viruses where thought to be a single genetic entity - yet quasispecies theory predicts that the population as a whole is the target of selection during infection. This is important, as evolution is thus affected not only by high mutation rates but also by interactions between the mutants, which can be positive or negative, and the breadth of the mutant spectrum can determine the behaviour of the population. For example, viral mutants that have higherfidelity polymerases (and thus populations with reduced heterogeneity) are generally attenuated in vivo and can have altered tissue tropism. In the case of poliovirus, these effects have been shown to be reversed by increasing the breadth of the mutant spectrum using chemical mutagenesis, evidencing positive interactions within the quasispecies ${ }^{5,6}$. On the other hand, mutant spectra can also suppress more fit genomes within a population, and attenuated or non-pathogenic virus variants have been shown to attenuate disease caused by more virulent components of a quasispecies ${ }^{5,6}$. Another consequence of this heterogeneity is that drug-resistance mutations can pre-exist in the population before the onset of antiviral treatment, which should thus be initiated early, before the quasispecies has had a chance to diversify ${ }^{5}$. Quasispecies theory also predicts the existence of an error threshold beyond which an increase in mutation rates would lead to extinction of viral populations. This has been shown to occur in vitro upon treatment of a variety of viruses with mutagens, and is the basis of the antiviral approach known as lethal mutagenesis ${ }^{5}$, which is an active area of research. In short, existing as a constantly changing cloud of related but non-identical genomes gives RNA viruses a plasticity that is important in the complex environments encountered in a host, during transmission to different hosts, and in response to therapy, and should be taken into consideration when developing antiviral drug and vaccine strategies.

It is remarkable that Domingo's first experiments were carried out before the methods to reverse-transcribe, amplify and sequence nucleic acids were established, when only a very partial view of the quasispecies could be obtained. The advent of next-generation sequencing, including methods that reduce background error specifically developed for the analysis of viral populations, has revolutionized this field. These approaches provide an unprecedented view of the composition of RNA virus quasispecies from human, animal and plant samples. When combined with the use of model systems and clinical data, these approaches are beginning to enable virologists to link the molecular understanding of quasispecies behaviour obtained in vitro with in vivo (intra-host) and populationlevel (inter-host) responses. The viral quasispecies field thus reaches middle age clearly re-energized by recent technological developments, in a vibrant mood and full of ideas, such as using in-host viral population dynamics as predictors of disease outcome or response to therapy, or the rational design of safe and effective live attenuated vaccines ${ }^{7}$.

As noted by Eigen in the foreword to a recent book on viral quasispecies ${ }^{5}$, the idea of heterogeneous populations has historically been quite uncommon in biology. However, more recently, the quasispecies concept has also been connected to other settings in which heterogeneity exists, such as mutator bacterial strains, prion conformational heterogeneity and cellular heterogeneity within tumours, although whether these systems behave according to the theory of quasispecies remains unclear.

As with the establishment of molecular biology a couple of decades earlier, close collaboration between theoreticians and experimentalists was key for the application of the quasispecies theory to RNA viral populations. During a small, interdisciplinary meeting convened by Eigen, he heard the unpublished results of the Weissmann lab and subsequent discussions led to the notion that $\mathrm{Q} \beta$ population dynamics could be explained by his recent theory. That seed fell on fertile ground, and a new field aiming to understand RNA virus heterogeneity, evolution and their manifold implications, took off. In the era of instant communication, of 'bubbles' or 'echo chambers' in social and professional networks, when often the same researchers attend the same meetings over the course of a year to discuss the same issues, we risk failing to learn a lesson from viruses on the strengths that can be gained from a diverse population enabling complementary and critical interactions. Gathering discussion in small, forwardlooking meetings of intentionally disparate researchers, often from entirely distinct disciplines, around a common topic, can foster a 'blue sky' atmosphere and the sharing of unpublished data, thus catalysing the emergence of new ideas and collaborations that are essential for scientific advancement. Such meetings should not be forfeit as a luxury in the face of budgetary strictures.

Published online: 27 March 2018 https://doi.org/10.1038/s41564-018-0145-3

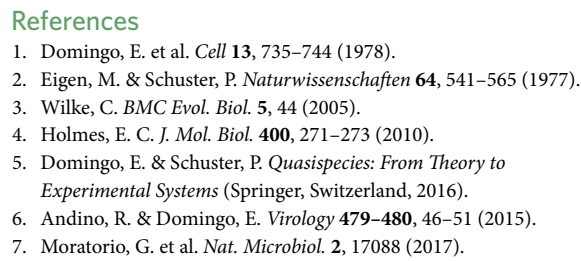

